\title{
Intelligent Multi-objective NSBGA-II Control of Power Converters in DC Microgrids
}

\author{
Vafamand, Arezoo; Vafamand, Navid; Zarei, Jafar; Razavi-Far, Roozbeh; Dragicevic, Tomislav
}

Published in:

IEEE Transactions on Industrial Electronics

Link to article, DOI:

10.1109/TIE.2020.3029483

Publication date:

2021

Document Version

Peer reviewed version

Link back to DTU Orbit

Citation $(A P A)$ :

Vafamand, A., Vafamand, N., Zarei, J., Razavi-Far, R., \& Dragicevic, T. (2021). Intelligent Multi-objective NSBGA-II Control of Power Converters in DC Microgrids. IEEE Transactions on Industrial Electronics, 68(11), 10806 - 10814. https://doi.org/10.1109/TIE.2020.3029483

\section{General rights}

Copyright and moral rights for the publications made accessible in the public portal are retained by the authors and/or other copyright owners and it is a condition of accessing publications that users recognise and abide by the legal requirements associated with these rights.

- Users may download and print one copy of any publication from the public portal for the purpose of private study or research.

- You may not further distribute the material or use it for any profit-making activity or commercial gain

- You may freely distribute the URL identifying the publication in the public portal 


\title{
Intelligent Multi-objective NSBGA-II Control of Power Converters in DC Microgrids
}

\author{
Arezoo Vafamand, Navid Vafamand, Jafar Zarei, Roozbeh Razavi-Far, and Tomislav Dragičević, \\ Senior Member, IEEE
}

\begin{abstract}
This paper develops a novel multi-objective controller to regulate the power converters of a class of direct current (DC) microgrids (MGs) connected to nonlinear constant power loads (CPLs) and linear resistive loads. The suggested control approach uses the nondominating sorting binary genetic algorithm (NSBGA-II) to directly design the on/off switching signal of the converters without using the pulse width modulation (PWM) technique. The multi-objective controller minimizes the tracking error of the DC bus voltage and at the same time tries to reduce the total number of switching actions. Thereby, the developed controller tracks the desired reference with a reduced converter switching action and power loss by using a proper Pareto solution. Moreover, by employing the NSBGA-II algorithm, it is feasible to involve the switching frequency in the design procedure to enhance the performance. Exploiting the binary genetic algorithm (BGA) instead of conventional GA, turns a continuous surface searching into a binary one, which not only makes it more compatible with the nature of the power converter control but also decreases the online computational burden. To illustrate the superiority of the proposed approach, real-time OPAL results are provided.
\end{abstract}

Index Terms-NGBGA-II, DC microgrid, Constant power load, Boost converter, Multi-objective control.

\section{INTRODUCTION}

Emerging direct current (DC) renewable sources, such as photovoltaic cells and wind turbines, energy storage systems such as batteries and the growing integration of electronic DC loads persuades considering DC MGs as a suitable and cost-effective power distribution topology [1]. Despite their control advantages such as circumventing frequency and reactive power regulation, DC MGs are often burdened by constant power loads (CPLs), which have destabilizing effects. With a growing share of tightly controlled loads, more and more loads in MGs act as CPLs. Such loads exhibit negative incremental resistance and may destabilize the overall system.

Thereby, the stability issue of the CPLs in the DC MGs is widely evaluated and several advanced control laws such as backstepping [2, 3], robust linear [4, 5], fuzzy-model-based [6], and model predictive control [7,8] are developed. In these control methods, overall DC MGs comprising switching power electronics converters and CPLs are represented by a state-space averaging model and then mainly controlled by the means of pulse-width modulation (PWM) techniques [9]. The switching

A. Vafamand is with Department of Electrical Engineering, K. N. Toosi University of Technology, Tehran, Iran. (email: a.vafamand@email.kntu.ac.ir)

N. Vafamand is with School of Electrical and Computer Engineering, Shiraz University, Shiraz, Iran (email: n.vafamand@shirazu.ac.ir)

J. Zarei is with the Department of Electrical and Electronics Engineering, Shiraz University of Technology, Shiraz, Iran; and, also with the Department of Electrical frequency in the PWM technique must be high enough to ensure the applicability of the averaged modeling technique, [10, 11]. However, neglecting the switching nature of power electronics converters limits the closed-loop performance and slows down the tracking demands $[9,12]$.

To bypass the PWM technique, the finite control set-model predictive control (FCS-MPC) method is exploited to directly design the pulsing signal of converters [9, 12]. Through this control approach, the continuous-time dynamics of the DC MG and switch model of a converter are used to predict the upcoming behavior of the converters in MGs. In contrast with the conventional MPC approaches [7, 8], which are established based on an averaged model of converters and use PWM, the FCS-MPCs bring about fast-tracking response by directly design the on/off gating signals of the converters and can handle the practical constraints of the MGs more effectively [13]. The FCS-MPC approach evaluates all future behavior the DC MG for all possible control inputs (i.e. on or off). Then, the best control input action, which results in the desired operation, is chosen. This approach has been widely considered for power electronics-based systems and control purposes [9, 12, 14-16]. However, FCS-MPC is based on the fact that all possible permutations for the on/off switch configurations of the converters must be enumerated online and the best solution is chosen based on exhaustive search. Therefore, by choosing a large predictive horizon, the number of permutations is increased exponentially. Thereby, the proposed approaches in $[9,12,14-16]$ use a one-step-ahead predictive control to decrease the computational burden at the expense of performance worsening. In [17], the results of one-step FCS are extended to a multi-step approach. To reduce the online computational burden of the multi-step FCS, the so-called sphere decoding algorithm (SDA) is utilized. In that technique, by ignoring the integer values of the switching control input, the optimal unconstrained control input law, which is the center of the sphere, is computed analytically. Then, the sequence of switching FCS inputs nearest to the unconstrained input law is chosen among all candidates. In [18], the SDA is improved to achieve a real-time implementation by projecting the unconstrained solution to a convex hull and reduce the number of candidates in the optimization problem. However, the SDA-based methods [17, 18] have some drawbacks in common. I) Finding the analytical unconstrained optimal solution is a difficult task for nonlinear systems [19]. Thereby, those approaches are not applicable for

and Computer Engineering, University of Windsor, Windsor, Canada (email: zarei@sutech.ac.ir)

R. Razavi-Far is with Department of Electrical and Computer Engineering, University of Windsor, Windsor, Canada. (email: roozbeh@uwindsor.ca)

T. Dragičević is with Department of Electrical Engineering, Technical University of Denmark, Lyngby, Denmark. (email: tomdr@elektro.dtu.dk) 
nonlinear DC MGs with CPLs. II) It is theoretically proved that the unconstrained analytical solution is not optimal for a constrained system and may fail to stabilize the closed-loop system [19]. Consequently, finding the nearest switching signal input to the unconstrained input signal is not an optimal solution and does not guarantee the closed-loop stability. III) Although some possible input candidates may be eliminated at each iteration of the optimization problem, it still needs to check many candidates to find the best one. Thereby, the online computational burden is high and dependent on the optimization problem's initial condition and the way of arranging the control input candidates. Moreover, in [9, 12, 14-18], the switching loss originated from the high number of switching actions is not involved.

Multi-objective evolutionary algorithms are introduced to treat the drawbacks of the FCS-MPC. Based on multi-objective optimization (MOO), it is feasible to increase the prediction horizon and at the same time keep the computational burden low, resulting in tracking performance improvement. This is achieved by the fact that intelligent metaheuristic algorithms use a limited number of random permutations among all possible solutions and then, they are evolved toward better solutions, at each of iteration. If a smaller number of initial guesses and a smaller number of iterations are chosen, the computational burden can be reduced. Moreover, if one of the objective functions is established to reduce the number of switching actions, the total switching power loss can be decreased. Up to now, several MOO and single-objective optimization (SOO) methods are suggested for power converters. For instance, in [20-23] the optimization methods are exploited to design more efficient converters. In [24, 25], the application of $\mathrm{MOO}$ and SOO to improve the droop control and power-sharing is studied. Moreover, the MOO and SOO are used to optimally design the stabilizing control law of the converters [26, 27]. In these control techniques, the MOO or SOO optimally tune the coefficients of the main controller, which generates a continuous duty cycle for the converters.

In this paper, a novel multi-objective controller is proposed to regulate the converters of DC MGs. The main distinguishing feature of the developed approach over the state-of-the-art results is that it uses the MOO to generate the pulsing signal of converters without the need for the PWM. Thereby, the suggested topology is different from state-of-the-art methods [26-30]. The proposed approach utilizes the non-dominating sorting binary genetic algorithm (NSBGA-II). Thorough the binary GA, surface searching is limited to binary zero and one control values that reduce the computational burden and generate the on/off pulsing signal of the converter. In this approach, a finite prediction horizon cost function is minimized, and a sequence of the pulsing signals is generated. Therefore, the suggested approach can be regarded as an extension of the conventional FSC-MPC [9, 12, 14-16], because it uses a larger prediction horizon and keeps the computational burden low. The multi-objective functions enable the reduction in the number of switching actions and consequently switching power loss. Moreover, as the PWM is avoided, it is feasible for the converter gates to switch with low frequency and involve the switching frequency into the cost function for design purposes. The considered DC MG supplies are both resistive loads and CPLs. Although the CPLs impose destabilizing effects on the DC MG, the suggested approach is able to stabilize the system and regulate DC bus voltage in the presence of disturbances. To illustrate the advantages of the NSBGA-II controller in terms of tracking performance and reduced switching actions, OPAL real-time (RT) experiments are carried out.

The organization of this paper is as follows: In Section II, the stand-alone DC MG feeding linear and nonlinear loads is presented. In Section III, the NSBGA-II algorithm is provided and the way of finding the optimum configuration of the gating signal of converters is discussed. In Section IV, the advantages and superiors of the proposed approach are discussed with details. In Section V, several experiments with different switching frequencies and Pareto solutions are provided, while the concluding remarks are given in Section VI.

\section{DC MICROGRID DYNAMIC}

A simplified circuit diagram of a standalone DC MG drawn in Fig. 1 comprises a DC source, constant power loads (CPLs), resistive loads, and energy storage units. This structure of DC MGs is widely deployed in several applications including more electric aircraft, ships, and automotive applications $[4,5]$.

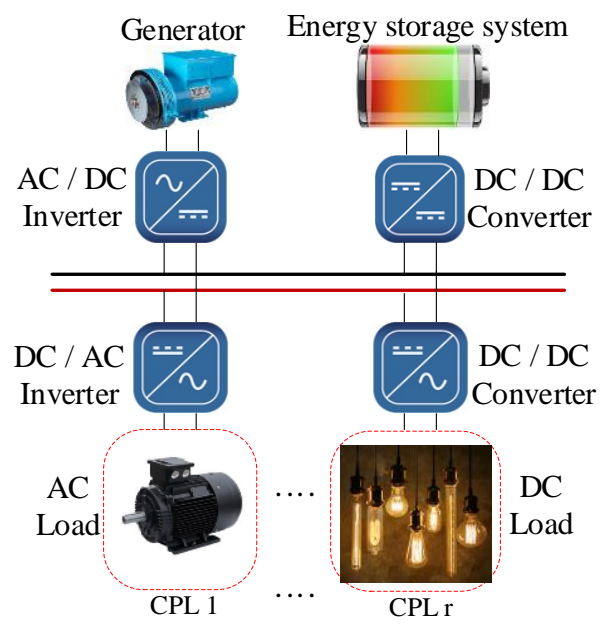

(a)

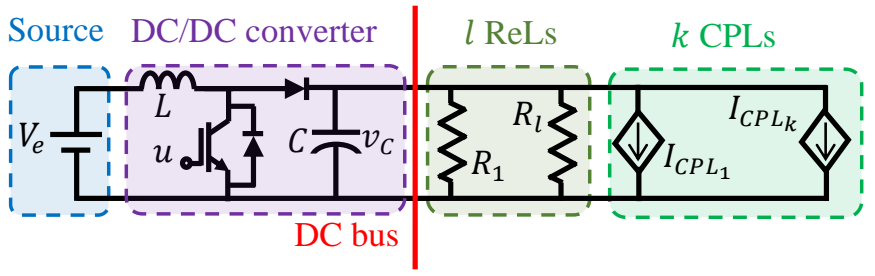

(b)

Fig. 1. The DC MG schematic. (a). detailed DC MG, (b). Simplified electric schematic.

Regarding the stability of the MGs with CPLs, they can be classified into three main groups, passive damping, active damping using auxiliary circuits, and source/CPL side active damping [31]. The last category deals with controlling the AC/DC inverters or DC/DC converters in series to the $\mathrm{AC}$ or DC sources, which is the main focus of this paper. Several sources in parallel in DC MGs need a suitable power-sharing strategy among them, thus raising the need for droop control technologies. In this paper, the problem of designing a novel switching control law for a standalone DC MG fed by only one DC source is investigated. However, the approach can easily be extended to multiple sources as well. Furthermore, the overall stability of DC MG can be guaranteed without any auxiliary energy storage systems. 
Therefore, the considered class of islanded DC MG with $l$ resistive loads (ReLs), and $r$ CPLs is supplied by only one DC source. The overall state-space representation of a such DC MG is obtained as [4]:

$$
\left\{\begin{array}{l}
L \frac{d i_{L}}{d t}=V_{e}-(1-u) v_{C} \\
C \frac{d v_{C}}{d t}=(1-u) i_{L}-\frac{v_{C}}{R_{1}}-\cdots-\frac{v_{C}}{R_{l}}-\frac{P_{C P l, 1}}{v_{C}}-\cdots-\frac{P_{C P l, r}}{v_{C}}
\end{array}\right.
$$

where $v_{C}$ and $i_{L}$ are the voltage of the DC link and the current of the converter, respectively, and are assumed to be measurable, $V_{e}$ is the constant voltage of the DC source, $R_{i}$ for $i=1, \ldots, l$ are the resistive loads, $P_{C P, i}$ for $i=1, \ldots, r$ are the power of the CPLs, and $u \in\{0,1\}$ is the switching control signal. $u=1$ corresponds to the conducting mode for the switch and $u=0$ corresponds to the nonconducting mode for the switch.

\section{NON-DOMINATING SORTING BINARY Genetic Algorithm} (NSBGA - II)

\section{a. Multi-objective optimization (MOO) problem}

In the considered DC MG with CPLs, there exist two objectives that should be optimized simultaneously. This problem is known as multi-objective optimization. The MOO can be regarded as the extension of conventional single-objective optimization. In the $\mathrm{SOO}$, one cost function is minimized to compute the optimal solution. However, in the MOO, there is not an optimal solution that minimizes all cost functions as there are some conflicts between them. Thereby, a trade-off, which is known as the Pareto optimal solution, between all costs has to be considered. More details of differences between the $\mathrm{SOO}$ and $\mathrm{MOO}$ are discussed in [33].

Pareto optimal set consists of different solutions, which are not dominated by the others and all of the solutions in the set do not have superiority over the other ones. Therefore, it is the designer decision to determine the weight value of the cost functions to sacrifice some of the cost functions to gain the other ones. Consider the following MOO with respect to the vector $x$ :

$$
\min J(x)=\left[J_{1}(x), J_{2}(x), \ldots, J_{k}(x)\right]^{T}
$$

Subject to

$$
\begin{gathered}
Q_{j}(x)=0 \text { for } j=1, \ldots, m \\
H_{j} \leq 0 \text { for } j=1, \ldots, n
\end{gathered}
$$

The Pareto optimal solution $x^{*}$ is defined as follows:

$$
\begin{aligned}
& \forall i=1, \ldots, k ; \nexists \bar{x}: J_{i}(\bar{x}) \leq J_{i}\left(x^{*}\right) \\
& \exists i=1, \ldots, k ; \exists \bar{x}: J_{i}(\bar{x}) \leq J_{i}\left(x^{*}\right)
\end{aligned}
$$

The relations (5) and (6) mean that one can find a solution $\bar{x}$ for which at least one (but not all) of the cost functions has a smaller value than the Pareto solution. Therefore, Pareto optimality provides an infinite number of solutions that are non-dominated.

Among these solutions, the decision-maker must subjectively choose the best one based on the physical properties of the system and the trade-off solution. Mainly, there are two options for this: I) considering a pre-defined and single criterion for the trade-off so that the MOO automatically chooses a solution; and II) considering an interactive procedure through which the designer follows the decision process.

Several evolutionary algorithms are suggested to find the nondominated Pareto solutions for a MOO. Some of them are strength Pareto evolutionary algorithm (SPEA) [34, 35], niched Pareto genetic algorithm (NPGA) [36], and the improved version of nondominated sorting genetic algorithm (NSGA-II) [37]. Among these approaches, the NSGA-II shows the most effective technique to find a diverse set of solutions and calculating the solution near true Pareto optimality solutions [38, 39].

\section{b. Conventional non-dominating Sorting Genetic Algorithm} (NSGA-II)

The work-flow of the NSGA-II for the $t$-th iteration is as follows: Initially, based on $N_{\text {pop }}$ parent population $P_{t}$ and applying the mutation and crossover, $N_{\text {pop }}$ offspring population members $Q_{t}$ are created. Then, both members $P_{t}$ and $Q_{t}$ are augmented in a combined population $R_{t}$, as

$$
R_{t}=\left(\begin{array}{lll}
P_{t} & \cup & Q_{t}
\end{array}\right)
$$

The population $R_{t}$ is sorted into $r$ domination levels $F_{j}$ for $i=$ $1, \ldots, r$ based on the objective functions, as

$$
R_{t}=\bigcup_{j=1}^{r} F_{j}
$$

Then, the members of $R_{t}$ the are sorted based on the objectives and the so-called crowding distance [40] in the new population $P_{t+1}$. The individuals of the population $P_{t+1}$ is constructed sequentially based on the priority of the non-domination levels $F_{j}$ with smaller indices. This procedure is started from the members of the first level $F_{1}$ and continued to the next levels until the number of these individuals is greater than or equal to $N_{\text {pop }}$. Assume that the level $F_{j}$ is the latest level. All individuals of the levels $F_{1}$ to $F_{j-1}$ are placed into the $P_{t+1}$. However, if all individuals of the level $F_{j}$ are added to $P_{t+1}$, then the individuals' number of the $P_{t+1}$ will be greater than $N_{\text {pop }}$. So, it is necessary to choose some of the individuals in $F_{j}$. This is done by using the crowding distance technique which is dependent on the objective functions. The algorithm of NSGA-II is illustrated in Fig. 2. As can be seen in Fig. 2, the individuals of $P_{t+1}$ are filled based on all members of $F_{1}$ to $F_{j-1}$ and some of $F_{j}$. The algorithms of nondominated and crowding disturbance sorting methods are presented in [40].

\section{c. Non-dominating Sorting Binary Genetic Algorithm (NSBGA-II)}

In the GA algorithm, individuals can have any continuous values. However, since the goal is to find the on/off switching commands of the converter, the binary genetic algorithm (BGA) replaces the conventional GA algorithm, leading to the nondominated sorting binary genetic algorithm (NSBGA-II).

In the BGA, the individuals accept integer values. Therefore, by setting these values to zero and one, the switching commands of the power electronic gates are obtained. Moreover, because of the special selection of the solution candidates, for the case of highdimensional surface searching, the BGA algorithm outperforms the classical GA optimization technique and provides less computational burden than linear programming or nonlinear constrained optimization [41].

The individuals in the NSBGA-II are binary character strings, called chromosomes. Each of the individuals stands for a possible solution within a search space and the number of individuals or chromosomes is the population of the NSBGA-II. The candidate individuals propagate through a process of evolution and result in a new generation of individuals, as shown in Fig. 2. Each evolution numerically provides more optimum individuals, which are closer 
to the Pareto optimal solution. Finally, whenever the stopping criteria are met, the candidate individual with the highest fitness will be selected as the output of NSBGA-II. This output comprises the on/off switching signals to be applied to the converter.

The candidate individuals include the parents and offspring as is evident in (7). The offspring population is generated by exploiting the crossover operator and mutation operators. The crossover operator produces one or two offsprings by mixing two selected parents, and the mutation operator is considered to escape from the local minimum. The details of producing the initial population, selection, crossover, and mutation can be found in [42].

\section{d. Defining objective functions for the DC MG with CPLs}

To have a reliable control, two issues should be considered. The first consideration is the voltage regulation of the bus. The first objective function is chosen to minimize the errors of the DC bus voltage and current from their desired values in a finite horizon, as

$$
J_{1}(t)=\sum_{i=1}^{n}\left(\left|v_{d}(n)-\hat{v}_{c}(n)\right|+\left|i_{d}(n)-\hat{i}_{L}(n)\right|\right)
$$

where $n$ stands for the prediction horizon, $v_{d}(n)$ and $i_{d}(n)$ are the desired references; and, the $\hat{v}_{c}(n)$ and $\hat{i}_{L}(n)$ are the future values of the DC MG system states calculated by predicting system states via the discretized dynamics (1).

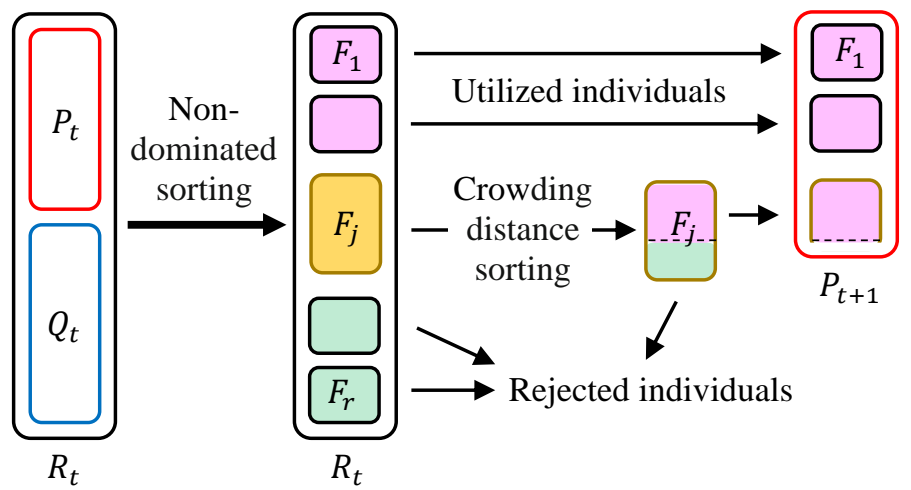

Fig. 2. Procedure producing the next population at each iteration.

On the other hand, the other critical issue in the design procedure is the power loss arisen by continuous switching actions of the power electronic devices. Therefore, it is desired to reduce the number of switching actions. This can be considered in two ways: I) reducing the bandwidth of the switching signal, and II) reducing the number of switching actions. The first option can be achieved by increasing the value of discretizing constant $T_{d}$. The second option is the minimize the variation of the switching actions among on/off situations. In this regard, the following objective function should be considered:

$$
J_{2}(t)=\sum_{i=1}^{n}(|\Delta u(n)|)
$$

where $\Delta u(n)=u(n+1)-u(n)$. The objective functions (9) are (10) have different effects on system performance. The former improves the transient and steady-state performance of the DC MG but voltage. The later reduces the power loss with the expense of degrading the voltage regulation performance. This compromise among the objective functions is treated by the NSBGA-II. By using the Pareto optimality approach, the designer is able to choose the weight of the objective functions (9) and (10) in constructing the switching signal.

\section{Closed-LOOP DC MG SYSTEM WITH NSBGA - II CONTROLLER}

In the state-of-the-art SOO [26-28, 30] and MOO [29] based control techniques, evolutionary algorithms are considered as an offline or online tuning tool. In other words, in [26-28, 30], a basic controller, which can be PI or PID is used to generate a continuous signal valued in the interval $u \in[0,1]$ and then applied to the PWM block to generate the switching signal; and evolutionary approaches are utilized to tune the gains and characteristics of the basic controller.

The proposed controller structure differs from the other conventional evolutionary-based controllers. In the proposed control approach, the evolutionary algorithm is used as the basic controller that produces integer values zero or one. Therefore, the output of the controller is directly applied to the converter without the need of the PWM. The overall structure of the DC MG system connected to the proposed NSBGA-II controller is depicted in Fig. 3. At each sampling instant, the current and voltage of the inductor and capacitor are measured. Then, for the given desired references and discretizing constant, the NSBGA-II produces a sequence of on/off switching commands.

The suggested approach can be extended to any DC MG systems with different topologies and loads. The state-space representation of the system is obtained and used instead of (1).

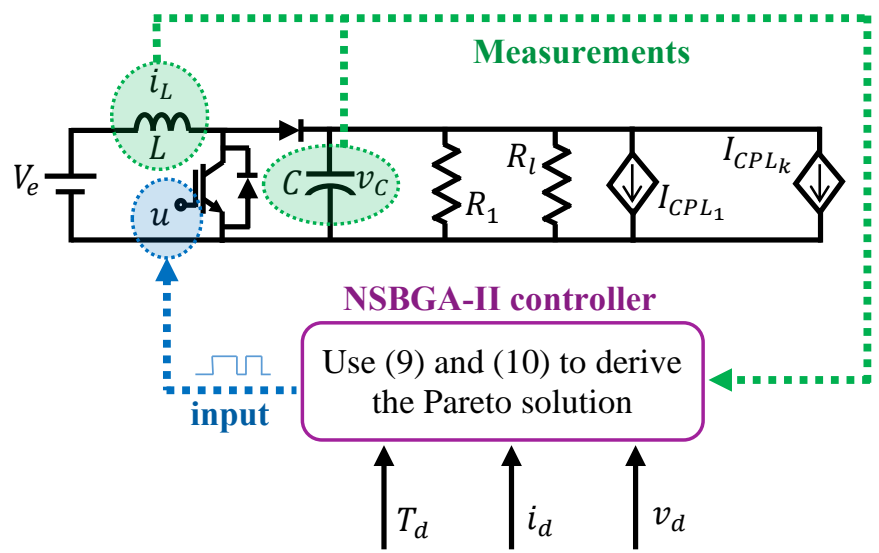

Fig. 3. The closed-loop system with the proposed NSBGA-II controller.

The proposed approach has several advantages and superiorities over the existing methods. The conventional control approaches use a heuristic algorithm to optimally tune the gains of an axillary controller, whose output is a smooth signal. Then, a PWM block is required to convert the smooth signal into the switching pulsation command for the converter. Thus, the overall implementation burden involves three parts of heuristic method, controller, and PWM block. On the other hand, the proposed approach removes the axillary controller and PWM, which reduces the implementation complexity. The developed approach is the general case of state-of-the-art FCS-MPC approaches. If the initial candidates of the NSBGA-II comprise all possible admissible candidates, the proposed approach is turned in the FCS-MPC. Letting $n$ prediction horizon to improve the closedloop performance mandates checking $2^{n}$ candidates in the FCS- 
MPC, which is time consuming. This drawback is handled by the proposed approach, as it needs a limited number of candidates to find the sub-optimum solution. Also, the best solution can be used as a candidate in the next optimization problem to improve the results gradually. Finally, the issue of switching frequency is involved in the design procedure. The switching frequency directly affects the DC-DC converter power loss and output voltage ripples. This issue is rarely considered in the existing design procedures. In the proposed approach, we can change the switching frequency online based on the system response to deal with the tradeoff between the transient and steady-state performances and the power loss.

The main drawback of heuristic algorithms is that they cannot assure the overall stability of the closed-loop system, because they use a numerical nature-inspired algorithm to solve the optimization problem. Though, it is shown that the heuristic-based control approaches successfully stabilize practical systems [4345].

\section{EXPERIMENTAL RESULTS}

To illustrate the applicability of the suggested approach, it is tested via the OPAL-RT hardware, as shown in Fig. 4. The controller and the DC MG system are implemented on a personal computer and the OPAL-RT hardware, respectively and they communicate by an ethernet LAN cable. The nominal values of the DC MG system parameters used in the NSBGA-II controller are given in Table I.

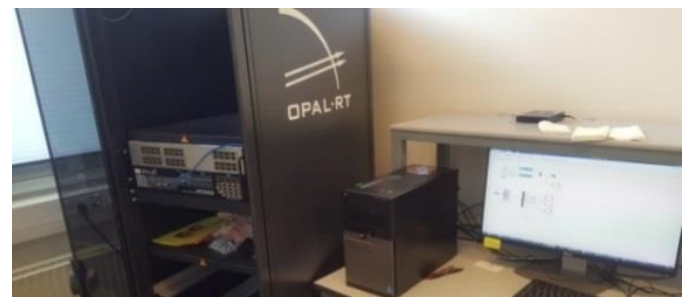

Fig. 4. The real-time setup for testing the control approach [7].

Table I: Nominal values of the DC MG

\begin{tabular}{ccc}
$R=30 \Omega$ & $C=500 \mu \mathrm{F}$ & $V_{e}=200 \mathrm{~V}$ \\
$P=300 \mathrm{~W}$ & $L=3 \mathrm{mH}$ & $v_{d}=400$ \\
\hline
\end{tabular}

Three scenarios are considered. In the first scenario, the effect of different Pareto solutions on the system performance and the number of switching variations are investigated. In the second scenario, the effect of the discretizing constant on the DC MG bus voltage is evaluated. And, in the third scenario, the effect of the load and desired voltage variations on the closed-loop system is investigated.

Furthermore the results of this paper are compared with FCSMPC [9]. This reference is considered to compare the results of this paper with, as it uses a predictive scheme with finite-set control without the need of the PWM technique. Though, the approach of [9] is modified for the case-study of this paper by updating its cost function and system state-space representation.

Scenario 1: In this scenario, for the fixed discretizing constant $T_{d}=0.01 \mathrm{~ms}$, and the prediction horizon $n=10$, the population number 10 , and the iteration number 10 , the NSBGA-II is performed.
A sample Pareto front is provided in Fig. 5, in which horizontal and vertical axes are the cost functions $J_{1}$ and $J_{2}$, respectively. From Fig. 5, one infers that the minimization of $J_{1}$ maximizes the $J_{2}$ and vice versa. To show the weights of the cost functions (9) and (10) three situations are considered and marked as S1, S2, and $\mathrm{S} 3$ with their weights values in Fig. 5. In the S1, the solution that minimizes the objective function (9) is used; The $\mathbf{S} 2$ is the middle point that simultaneously minimizes both $J_{1}$ and $J_{2}$ with almost the same percentage. And the $\mathrm{S} 3$ is the case that the $J_{2}$ is minimized.

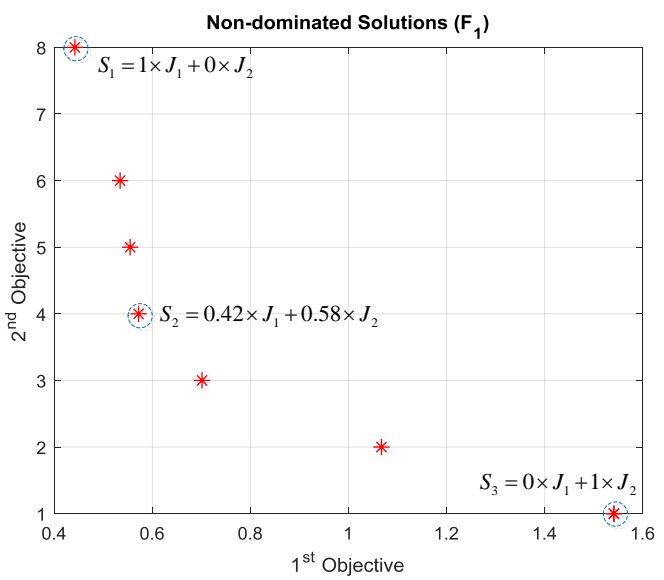

Fig. 5. The Pareto front for the first step of designing the switching control law in Scenario 1.

Fig. 6 shows the real-time response and control input of the DC MG system based on the three Pareto S1, S2, and S3 and the FCSMPC [9]. Fig. 6 Reveals that three Pareto solutions can stabilize the system and keep the DC bus voltage near the desired reference $v_{d}=400 \mathrm{~V}$. However, the best performance is achieved for S1, in which the response error is minimized. The smallest switching variations belong to the $\mathrm{S} 3$, which minimizes the control input changes. On the other hand, for the S2, the voltage output tracks the desired voltage reference and the total number of on/off changes is reduced.

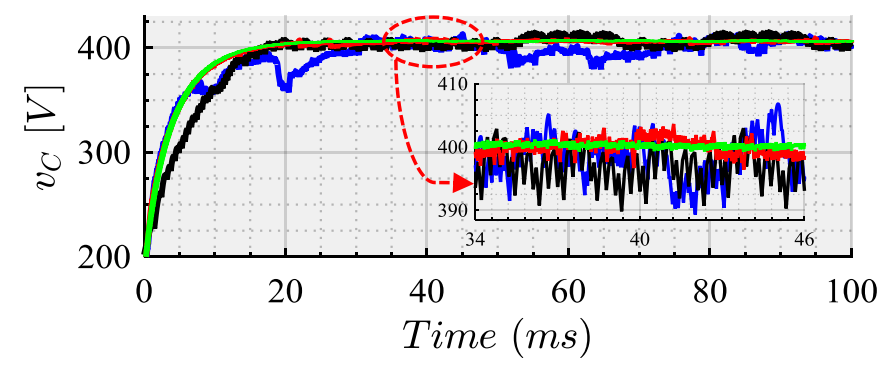

(a)

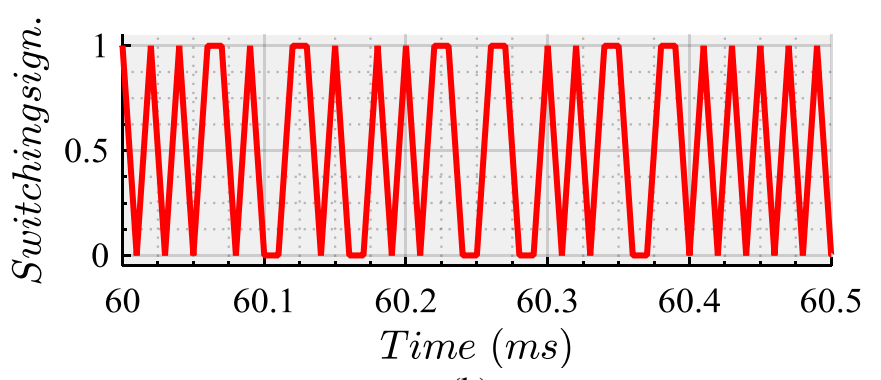

(b) 


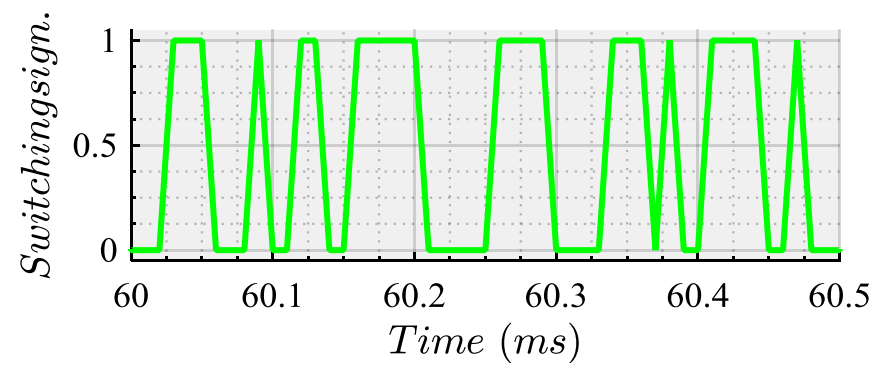

(c)

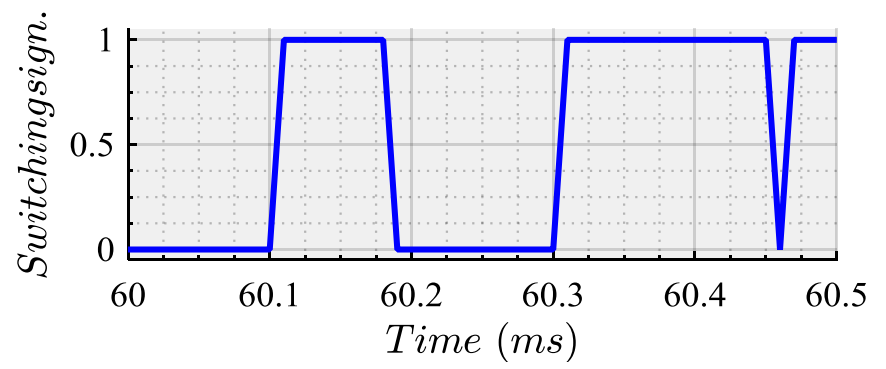

(d)

Fig. 6. The closed-loop system response and switching control input with $T_{d}=0.01 \mathrm{~ms}$ ( $\mathrm{S} 1$ is the red line, $\mathrm{S} 2$ is the green line, $\mathrm{S} 3$ is the blue

line, and the modified version of [9] is the black line): (a). DC bus voltage, (b)-(d). Control inputs.

Moreover, S1 and S2 of the proposed approach have a faster reaching-time and the smaller voltage ripple than the modified approach of [9]. This is mainly caused by the fact that the proposed approach uses a larger prediction horizon than [9].

Scenario 2: In Scenario 2, the proposed approach with the parameters given in Scenario 1 and the fixed discretizing constant $T_{d}=0.1 \mathrm{~ms}$ is applied to the DC MG converter. As can be seen in Fig. 7, the best performance is obtained by $\mathrm{S} 1$, followed by $\mathrm{S} 2$. Moreover, the Pareto solutions S2 and S3 gain benefit from low switching variations than the $\mathrm{S} 1$.

To better compare the results of Scenarios 1 and 2 Table II is provided.

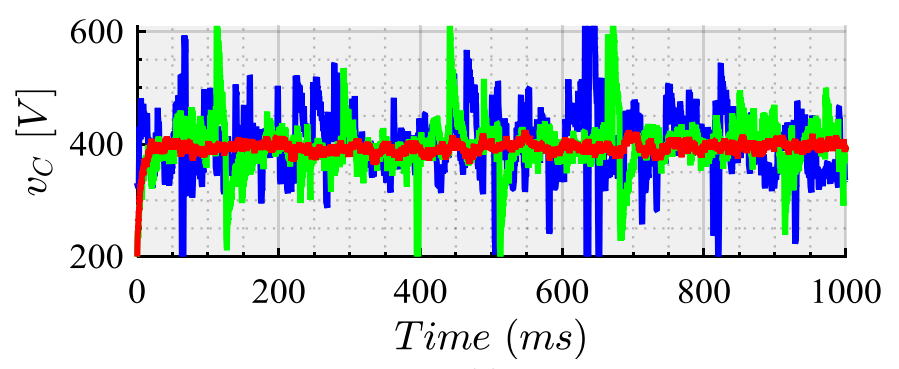

(a)

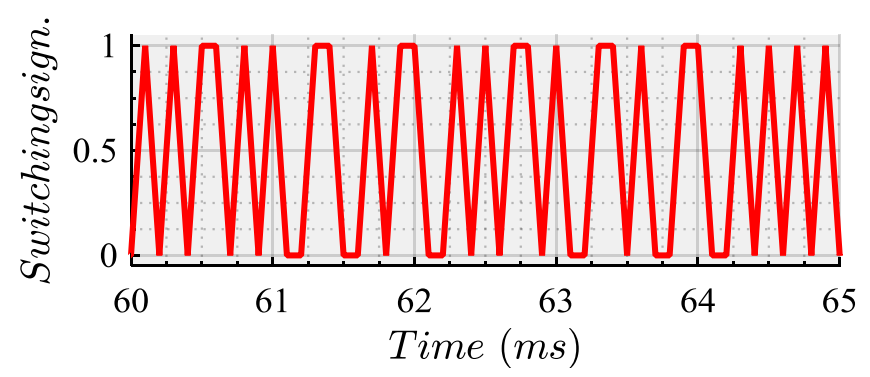

(b)

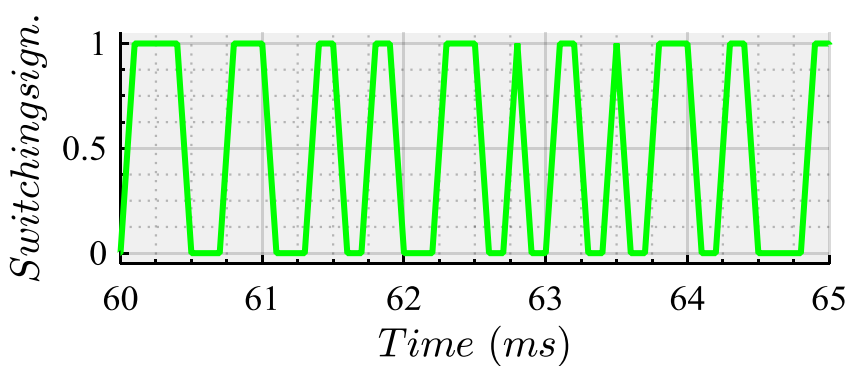

(c)

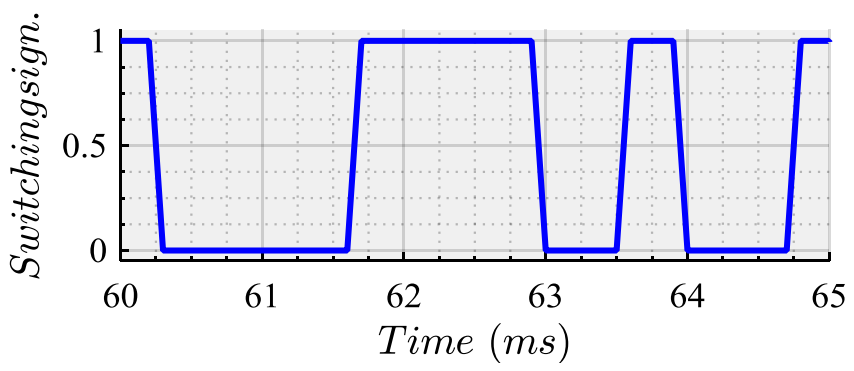

(d)

Fig. 7. The closed-loop system response and switching control input with $T_{d}=0.1 \mathrm{~ms}$ ( $\mathrm{S} 1$ is the red line, $\mathrm{S} 2$ is the green line, and S3 is the blue line): (a). DC bus voltage, (b)-(d). Control inputs.

Table II contains reaching time, norm 2 of the tracking error, norm $\infty$ of the steady-state tracking error, and the total number of switching variations. Table II reveals that for the high switching frequency of Scenario 1, the Pareto solutions result in almost the same tracking performance, but the number of switching variations can be reduced. On the other hand, by choosing lower switching frequency, the tracking error for the cases that the second objective function is more minimized may be degraded. From another point of view, for the low switching frequency, the reaching-time and the steady-state performances are degraded and the DC bus voltage ripple is increased. This shows the necessity of optimally choose the switching frequency and Pareto solutions for the power converters.

Table II: performance comparison of different Pareto cases.

\begin{tabular}{|c|c|c|c|c|c|}
\hline & & $\begin{array}{c}\text { Reaching } \\
\text { time } \\
(80 \%) \\
\end{array}$ & $\begin{array}{c}\text { Tracking } \\
\text { error } \\
\text { Norm } 2 \\
\end{array}$ & $\begin{array}{l}\text { Steady- } \\
\text { state error } \\
\text { Norm } \infty\end{array}$ & $\begin{array}{c}\text { Switching } \\
\text { changes } \\
\text { number }\end{array}$ \\
\hline \multirow{3}{*}{ 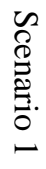 } & S1 & 16.1705 & $2.84 e+03$ & 1.2104 & 7337 \\
\hline & $\mathrm{S} 2$ & 16.1913 & $2.94 e+03$ & 2.1413 & 3628 \\
\hline & S3 & 16.3491 & $3.13 e+03$ & 7.8676 & 1267 \\
\hline \multirow{3}{*}{ 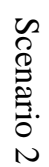 } & S1 & 29.654 & $2.16 e+03$ & 12.8466 & 735 \\
\hline & $\mathrm{S} 2$ & 29.838 & $5.70 \mathrm{e}+03$ & 95.9057 & 358 \\
\hline & S3 & 33.845 & $6.35 e+03$ & 108.7434 & 114 \\
\hline
\end{tabular}

Scenario 3: The goal of this scenario is to evaluate the performance of the proposed approach in the load and desired voltage changes. The Pareto solution S1 of Scenario 1 is considered in this scenario. It is assumed that the CPL power and the resistive load vary as given in Fig. 8. 


\section{CONCLUSION}

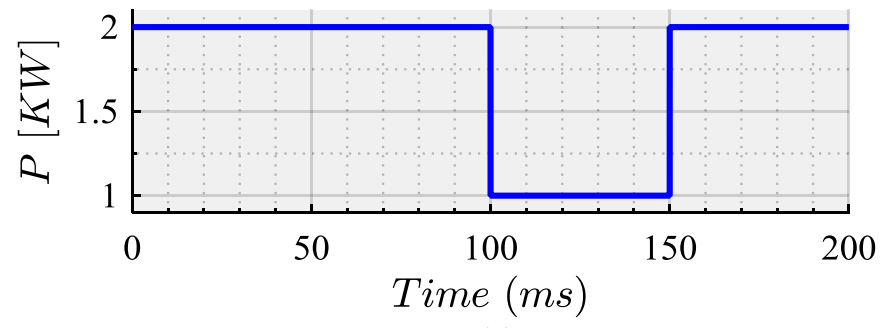

(a)

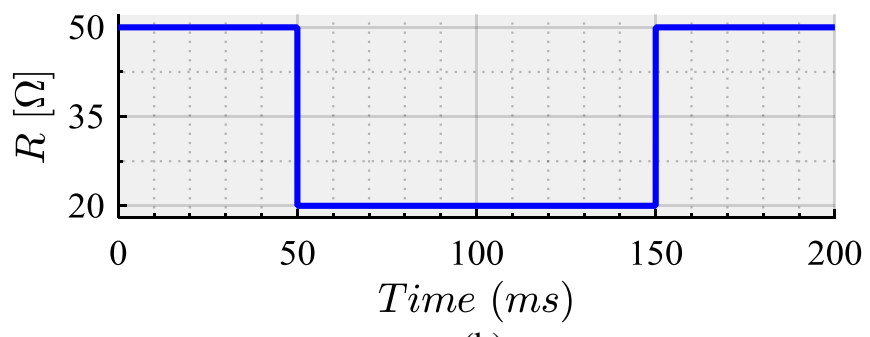

(b)

Fig. 8. The load variation. (a). The CPL and (b) The resistive load.

However, the nominal values $P=2 \mathrm{KW}$ and $R=50 \Omega$ are used in the controller design and so, the load changes are not measured and can be considered as uncertainty. The DC bus voltage is show in Fig. 9. As can be seen in Fig. 9, the proposed approach is able to reject the variations of loads. By comparing the instances 50 and $100 \mathrm{~ms}$, one observes that the resistive load variation has more effect than the CPL on the DC bus voltage. Also, whenever both CPL and resistive load changes, the proposed approach stabilizes the system slower than the case of sole loads changes.

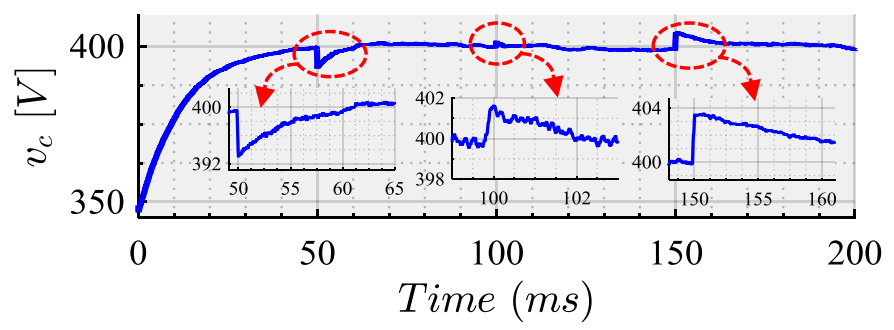

Fig. 9. The closed-loop system response to the load variation.

Fig. 10 illustrates the response of the closed-loop system to the desired voltage level changes. Fig. 10 reveals that the controller needs $20 \mathrm{~ms}$ to completely regulate the DC bus voltage with respect to the step changes.

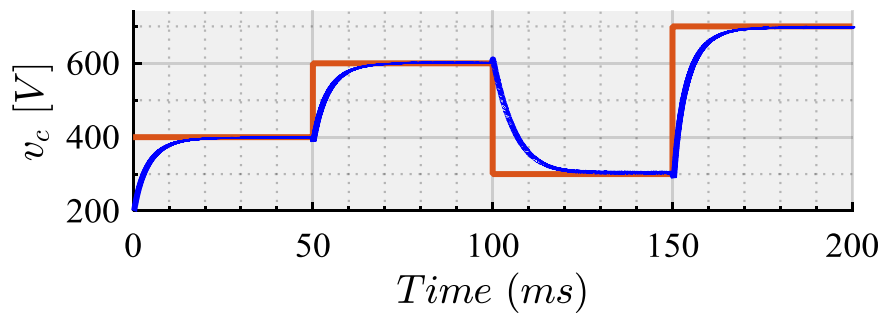

Fig. 10. The closed-loop system response to the desired voltage variation.
In this paper, a novel approach to directly controller the boost converters of the DC MGs supplying constant power and resistive loads is presented. The proposed approach utilizes the BGA to design the on/off switching signal of the converters. The proposed approach outperforms the finite control set method in the points of view of transient performance and low computational burden of the large prediction horizon. The proposed approach is experimentally tested on a DC MG setup and the obtained results verified the effectiveness of the suggested approach in tracking the desired reference. Therefore, the settling time of the system responses is improved. For future work, the following improvements are suggested. I) employing the other heuristic and metaheuristic optimization techniques. II) Analyzing the stability and robustness of the closed-loop theoretically. III) proposing a systematic approach to choose the desired Pareto solution to offer a fast DC voltage tracking convergence at the transient stage and low power loss at the steady-state phase. IV) evaluating the power loss and maximum ripple based on the proposed approach and studying those relations with the switching frequency.

\section{REFERENCES}

[1] S. Yousefizadeh, J. D. Bendtsen, N. Vafamand, M. H. Khooban, F. Blaabjerg, and T. Dragičević, "Tracking control for a DC microgrid feeding uncertain loads in more electric aircraft: Adaptive backstepping approach," IEEE Transactions on Industrial Electronics, vol. 66, no. 7, pp. 5644-5652, 2018.

[2] G. Sulligoi, D. Bosich, G. Giadrossi, L. Zhu, M. Cupelli, and A. Monti, "Multiconverter medium voltage DC power systems on ships: Constantpower loads instability solution using linearization via state feedback control," IEEE Transactions on Smart Grid, vol. 5, no. 5, pp. 2543-2552, 2014.

[3] Q. Xu, C. Zhang, C. Wen, and P. Wang, "A novel composite nonlinear controller for stabilization of constant power load in DC microgrid," IEEE Transactions on Smart Grid, vol. 10, no. 1, pp. 752-761, 2017.

[4] L. Herrera, W. Zhang, and J. Wang, "Stability analysis and controller design of DC microgrids with constant power loads," IEEE Transactions on Smart Grid, vol. 8, no. 2, pp. 881-888, 2015.

[5] M. A. Kardan et al., "Improved stabilization of nonlinear DC microgrids: Cubature Kalman filter approach," IEEE Transactions on Industry Applications, vol. 54, no. 5, pp. 5104-5112, 2018.

[6] M. M. Mardani, N. Vafamand, M. H. Khooban, T. Dragičević, and F. Blaabjerg, "Design of quadratic D-stable fuzzy controller for DC microgrids with multiple CPLs," IEEE Transactions on Industrial Electronics, vol. 66, no. 6, pp. 4805-4812, 2018.

[7] N. Vafamand, M. H. Khooban, T. Dragičević, and F. Blaabjerg, "Networked fuzzy predictive control of power buffers for dynamic stabilization of DC microgrids," IEEE Transactions on Industrial Electronics, vol. 66, no. 2, pp. 1356-1362, 2018.

[8] N. Vafamand, S. Yousefizadeh, M. H. Khooban, J. D. Bendtsen, and T. Dragičević, "Adaptive TS fuzzy-based MPC for DC microgrids with dynamic CPLs: Nonlinear power observer approach," IEEE Systems Journal, vol. 13, no. 3, pp. 3203-3210, 2018.

[9] M. Novak, U. M. Nyman, T. Dragicevic, and F. Blaabjerg, "Analytical design and performance validation of finite set MPC regulated power converters," IEEE Transactions on Industrial Electronics, vol. 66, no. 3, pp. 2004-2014, 2018.

[10] A. Ayachit and M. K. Kazimierczuk, "Averaged small-signal model of PWM DC-DC converters in CCM including switching power loss," IEEE Transactions on Circuits and Systems II: Express Briefs, vol. 66, no. 2, pp. 262-266, 2018

[11] Y. Xiong, S. Sun, H. Jia, P. Shea, and Z. J. Shen, "New physical insights on power MOSFET switching losses," IEEE Transactions on Power Electronics, vol. 24, no. 2, pp. 525-531, 2009.

[12] J. Rodriguez et al., "State of the art of finite control set model predictive control in power electronics," IEEE Transactions on Industrial Informatics, vol. 9, no. 2, pp. 1003-1016, 2012.

[13] T. Dragičević, "Dynamic stabilization of DC microgrids with predictive control of point-of-load converters," IEEE Transactions on Power 
Electronics, vol. 33, no. 12, pp. 10872-10884, 2018.

[14] T. Dragičević and M. Novak, "Weighting factor design in model predictive control of power electronic converters: An artificial neural network approach," IEEE Transactions on Industrial Electronics, vol. 66, no. 11, pp. 8870-8880, 2018.

[15] J. Rodriguez et al., "Predictive control of three-phase inverter," Electronics letters, vol. 40, no. 9, pp. 561-563, 2004.

[16] S. Vazquez, P. Acuna, R. P. Aguilera, J. Pou, J. I. Leon, and L. G. Franquelo, "DC-Link Voltage Balancing Strategy Based on Optimal Switching Sequences Model Predictive Control for Single-Phase H-NPC Converters," IEEE Transactions on Industrial Electronics, 2019.

[17] T. Geyer and D. E. Quevedo, "Multistep finite control set model predictive control for power electronics," IEEE Transactions on power electronics, vol. 29, no. 12, pp. 6836-6846, 2014.

[18] P. Karamanakos, T. Geyer, and R. P. Aguilera, "Long-horizon direct model predictive control: Modified sphere decoding for transient operation," IEEE Transactions on Industry Applications, vol. 54, no. 6, pp. 6060-6070, 2018.

[19] D. E. Kirk, Optimal control theory: an introduction. Courier Corporation, 2004.

[20] R. M. Burkart and J. W. Kolar, "Comparative \$leta \$-\$rho \$-\$isigma \$ Pareto Optimization of $\mathrm{Si}$ and $\mathrm{SiC}$ Multilevel Dual-Active-Bridge Topologies With Wide Input Voltage Range," IEEE Transactions on Power Electronics, vol. 32, no. 7, pp. 5258-5270, 2016.

[21] C. Larouci, M. Boukhnifer, and A. Chaibet, "Design of power converters by optimization under multiphysic constraints: application to a two-time-scale AC/DC-DC converter," IEEE Transactions on Industrial Electronics, vol. 57, no. 11, pp. 3746-3753, 2010.

[22] A. Stupar, T. Friedli, J. Minibock, and J. W. Kolar, "Towards a 99\% efficient three-phase buck-type PFC rectifier for 400-V DC distribution systems," IEEE Transactions on Power Electronics, vol. 27, no. 4, pp. 1732-1744, 2011.

[23] A. Stupar, T. McRae, N. Vukadinović, A. Prodić, and J. A. Taylor, "MultiObjective Optimization of Multi-Level DC-DC Converters Using Geometric Programming," IEEE Transactions on Power Electronics, vol. 34, no. 12, pp. 11912-11939, 2019.

[24] A. M. Dissanayake and N. C. Ekneligoda, "Multi-Objective Optimization of Droop Controlled Distributed Generators in DC Microgrids," IEEE Transactions on Industrial Informatics, 2019.

[25] K. Yu, Q. Ai, S. Wang, J. Ni, and T. Lv, "Analysis and optimization of droop controller for microgrid system based on small-signal dynamic model," IEEE Transactions on Smart Grid, vol. 7, no. 2, pp. 695-705, 2015.

[26] M. J. Neath, A. K. Swain, U. K. Madawala, and D. J. Thrimawithana, "An optimal PID controller for a bidirectional inductive power transfer system using multiobjective genetic algorithm," IEEE Transactions on Power Electronics, vol. 29, no. 3, pp. 1523-1531, 2013.

[27] K. Sundareswaran and V. Sreedevi, "Boost converter controller design using queen-bee-assisted GA," IEEE Transactions on industrial electronics, vol. 56, no. 3, pp. 778-783, 2008.

[28] S. F. Faisal, A. R. Beig, and S. Thomas, "Time Domain Particle Swarm Optimization of PI Controllers for Bidirectional VSC HVDC Light System," Energies, vol. 13, no. 4, p. 866, 2020.

[29] K. Rouzbehi, M. Baa Wafaa, and E. Rakhshani, "An Imperialist Competitive Algorithm-Based Multi-Objective Optimization for Voltage Source Converter High-Voltage Direct Current Stations Control in Multi-Terminal HVDC Grids," Electric Power Components and Systems, vol. 47, no. 4-5, pp. 316-328, 2019.

[30] G. M. Vargas Gil, L. Lima Rodrigues, R. S. Inomoto, A. J. Sguarezi, and R. Machado Monaro, "Weighted-PSO Applied to Tune Sliding Mode Plus PI Controller Applied to a Boost Converter in a PV System," Energies, vol. 12, no. 5, p. 864, 2019.

[31] E. Hossain, "Addressing instability issues in microgrids caused by constant power loads using energy storage systems," 2016.

[32] G. Akudibillah, A. Pandey, and J. Medlock, "Optimal control for HIV treatment," 2018.

[33] K. Deb, Multi-objective optimization using evolutionary algorithms. John Wiley \& Sons, 2001.

[34] S. Jiang and S. Yang, "A strength Pareto evolutionary algorithm based on reference direction for multiobjective and many-objective optimization," IEEE Transactions on Evolutionary Computation, vol. 21, no. 3, pp. 329346, 2017.

[35] A. H. Naghshbandy and A. Faraji, "Coordinated design of PSS and unified power flow controller using the combination of CWT and Prony methods with the help of SPEA II multi-objective optimisation algorithm," IET Generation, Transmission \& Distribution, vol. 13, no. 21, pp. 4900-4909, 2019.
[36] K. Chakkarapani, T. Thangavelu, K. Dharmalingam, and P. Thandavarayan, "Multiobjective design optimization and analysis of magnetic flux distribution for slotless permanent magnet brushless DC motor using evolutionary algorithms," Journal of Magnetism and Magnetic Materials, vol. 476, pp. 524-537, 2019.

[37] L. Xiaoqing, D. Haiying, L. Hongwei, L. Mingxue, and S. Zhiqiang, "Optimization Control of Front-End Speed Regulation (FESR) Wind Turbine Based on Improved NSGA-II," IEEE Access, vol. 7, pp. 45583-45593, 2019.

[38] M. E. Hamidi and R. M. Chabanloo, "Optimal Allocation of Distributed Generation With Optimal Sizing of Fault Current Limiter to Reduce the Impact on Distribution Networks Using NSGA-II," IEEE Systems Journal, vol. 13, no. 2, pp. 1714-1724, 2018.

[39] U. Singh and S. N. Singh, "Optimal feature selection via NSGA-II for power quality disturbances classification," IEEE Transactions on Industrial Informatics, vol. 14, no. 7, pp. 2994-3002, 2017.

[40] K. Deb, A. Pratap, S. Agarwal, and T. Meyarivan, "A fast and elitist multiobjective genetic algorithm: NSGA-II," IEEE transactions on evolutionary computation, vol. 6, no. 2, pp. 182-197, 2002.

[41] D. Rerkpreedapong, A. Hasanovic, and A. Feliachi, "Robust load frequency control using genetic algorithms and linear matrix inequalities," IEEE Transactions on Power Systems, vol. 18, no. 2, pp. 855-861, 2003.

[42] S. Sivanandam and S. Deepa, "Genetic algorithms," in Introduction to genetic algorithms: Springer, 2008, pp. 15-37.

[43] P. R. U. Guazzelli, W. C. de Andrade Pereira, C. M. R. de Oliveira, A. G. de Castro, and M. L. de Aguiar, "Weighting factors optimization of predictive torque control of induction motor by multiobjective genetic algorithm," IEEE Transactions on Power Electronics, vol. 34, no. 7, pp. 6628-6638, 2018

[44] U. Lee, S. Park, and I. Lee, "Robust design optimization (RDO) of thermoelectric generator system using non-dominated sorting genetic algorithm II (NSGA-II)," Energy, vol. 196, p. 117090, 2020.

[45] S. Wang, D. Zhao, J. Yuan, H. Li, and Y. Gao, "Application of NSGA-II Algorithm for fault diagnosis in power system," Electric Power Systems Research, vol. 175, p. 105893, 2019.

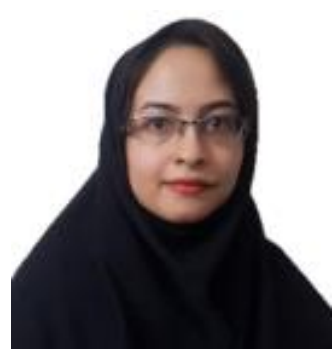

Arezoo Vafamand was born in Shiraz, Iran in 1993. She received the B.Sc degree in control engineering from Shiraz University of Technology, Shiraz, Iran in 2015 and M.Sc degree in control engineering from Khajeh Nasir Toosi University of Technology, Tehran, Iran in 2019. Her research interest includes model predictive control, TS fuzzy systems, and Intelligent algorithms.

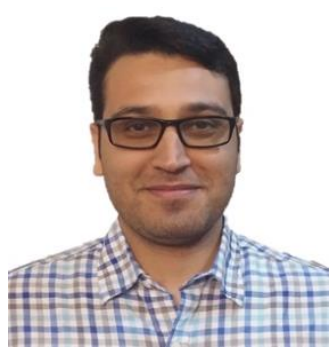

Navid Vafamand received his B.Sc. degree in electrical engineering and M.Sc. degree in control engineering form Shiraz University of Technology, Iran, in 2012 and 2014, respectively, and Ph. D. in control engineering at Shiraz University, Shiraz, Iran, in 2019. Currently, he serves as a research assistance at Shiraz University. He was a Ph.D. Visiting student with the Department of Energy Technology, Aalborg University, Denmark, from 2017 to 2018. Dr. Vafamand is the co-author of more than 80 international conference and journal papers and two chapter-books and an active reviewer in several journals. His main research interests include Takagi-Sugeno (TS) fuzzy systems, linear parameter varying (LPV) models, predictive control, and DC microgrids. 


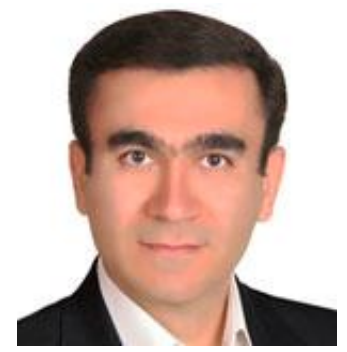

Jafar Zarei (M'16) received his B.S. degree from Shiraz University, Shiraz, Iran, in 2002, and the M.S. and Ph.D. degrees from the Iran University of Science and Technology, Tehran, Iran, in 2005, and 2011, respectively, all in Electrical Engineering. Since 2011, he has been with the Shiraz University of Technology, Shiraz, where he is currently an Associate Professor with the faculty of Electrical and Electronics Engineering. He has been Research Associate in the Faculty of Engineering at the University of Windsor, Canada since August 2019. His current research interests include on the theoretical side, estimation theory, robust control, analysis and control of networked control systems (fuzzy, Markovian jump models), cyber-physical systems, fault detection and isolation, fault tolerant control, analysis and control via linear matrix inequality optimization techniques, and Lyapunov methods. On the application side he is mainly interested in fault detection of induction motors

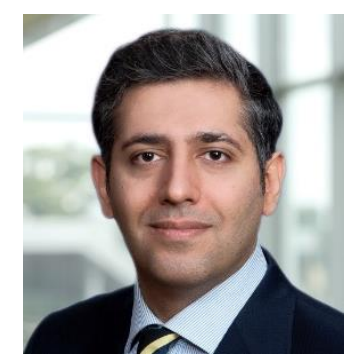

Roozbeh Razavi-Far (S'08-M'17SM'19) received the B.Sc. degree in Electrical Engineering in 2001, the M.Sc. and Ph.D. degrees from Amirkabir University of Technology, Tehran Polytechnic, in 2004 and 2009, respectively, and achieved a second Ph.D. degree in Nuclear Engineering from Politecnico di Milano, in 2011. He was a postdoctoral fellow at the Universitl'e libre de Bruxelles, Belgium and an NSERC postdoctoral fellow at NRCan, Canada. He is currently with the Faculty of Engineering at University of Windsor. He is an Adjunct Professor with the Department of Electrical and Computer Engineering and Cross-Appointed Professor with the School of Computer Science at University of Windsor. His research focuses on machine learning, big data analytics, computational intelligence, cybernetics, diagnostics and prognostics of cyber-physical systems. He has served as an Associate Editor for Neurocomputing and KES journals. He is the Chapter Chair of IEEE Computational Intelligence, and Systems, Man and Cybernetics Societies, Windsor section.

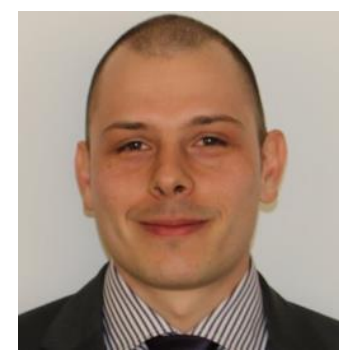

Tomislav Dragičević (S'09-M'13SM'17) received the M.Sc. degree and the industrial Ph.D. degrees in electrical engineering from the Faculty of Electrical Engineering, University of Zagreb, Zagreb, Croatia, in 2009 and 2013, respectively.,From 2013 to 2016, he was a Postdoctoral Research Associate with Aalborg University, Denmark, where he was an Associate Professor from 2016 to 2020. He was a Guest Professor at Nottingham University, U.K., during Spring/Summer of 2018. Since 2020, he has been a
Professor with the Technical University of Denmark. His research interests include design and control of microgrids, and application of advanced modeling and control concepts to power electronic systems. He has authored and coauthored more than 200 technical papers (more than 100 of them are published in international journals, mostly in IEEE), eight book chapters, and a book in the field.,Dr. Dragičević is an Associate Editor of the IEEE Transactions on Industrial Electronics, IEEE Transactions on Power Electronics, IEEE Journal of Emerging and Selected Topics in Power Electronics, and IEEE Industrial Electronics Magazine. He was a recipient of the Končar Prize for the Best Industrial Ph.D. thesis in Croatia, and a Robert Mayer Energy Conservation Award. He is a winner of Alexander van Humboldt fellowship for experienced researchers. 